\title{
Spatial analyses of typhoid fever in Jiangsu province, People's Republic of China
}

Yue-Jia Cheng', Fen-Yang Tang ${ }^{2}$, Chang-Jun $\mathrm{Bao}^{2}$, Ye-Fei Zhu², Qi Liang ${ }^{2}$, Jian-Li Hu², Wen-Dong $\mathrm{Liu}^{2}$, Ying Wu², Kathleen H. Reilly ${ }^{3}$, Tong-Qian Shen ${ }^{1}$, Yang Zhao', Zhi-Hang Peng ${ }^{1}$, Rong-Bin Yu ${ }^{1}$, Hua Wang ${ }^{2}$, Hong-Bing Shen ${ }^{1}$, Feng Chen ${ }^{1}$

${ }^{1}$ Department of Epidemiology and Biostatistics, School of Public Health, Nanjing Medical University, Nanjing, 211166, People's Republic of China: ${ }^{2} J i a n g s u$ Province Center for Disease Control and Prevention, Nanjing, 210009, People's Republic of China; ${ }^{3}$ Tulane University Health Sciences Center, School of Public Health and Tropical Medicine, 1440 Canal Street, New Orleans, LA 70112, USA

\begin{abstract}
An analysis of the geographical distribution of typhoid incidence rates, based on various statistical approaches such as trend surface, spatial autocorrelation, spatial correlation and spatial regression, was carried out at the county level in Jiangsu province, People's Republic of China. Temperature, moisture content, proximity to water bodies and the normalized difference vegetation index in the autumn were the four underlying factors found to contribute the most to the development of the epidemic. Typhoid infection was most severe in the south-eastern region of Jiangsu and a significant hotspot with high positive autocorrelation was detected in Taicang county in the south-east of the province. To improve the typhoid situation, intervention efforts should be concentrated in the south-eastern region of the province, targeting the hotspot and include reduction of lake pollution.
\end{abstract}

Keywords: geographical information systems, typhoid fever, trend surface analysis, spatial analysis, spatial regression, People's Republic of China.

\section{Introduction}

Typhoid fever is a life-threatening illness caused by the bacterium Salmonella typhi. Historically, in the People's Republic of China (P.R. China), this infection caused high levels of morbidity and mortality up to the 1990s due to poor sanitation and faecal contamination of water and food (Crump and Mintz, 2010; Dong et al., 2010). However, the implementation of the Ninth and Tenth Five-Year Plans (from 1996 to 2006) helped reducing transmission of epidemics by providing financial resources to improve sanitation. Although this was helpful for Jiangsu province, a prevalence fluctuation of typhoid fever occurred around 2004 (Dong et al., 2006). Studies show that sewage contamination, poor individual hygiene prac-

\footnotetext{
Corresponding author:

Zhi-Hang Peng

Department of Epidemiology and Biostatistics

School of Public Health, Nanjing Medical University

818 East Tianyuan Road

Jiangning District, Nanjing 211166, People's Republic of China

Tel. +86 258686 8436; Fax +86 2586868499

E-mail: zhihangpeng@njmu.edu.cn
}

tices and other attributes (e.g. foreign travel), have destabilised the typhoid situation in recent years (Ochiai et al., 2008). Infections occur disproportionately among children and students as the $S$. typhi bacteria commonly show strain variations leading to higher risk for those with less developed immunity against the wide variations of infective agents, i.e. the younger population (Bao et al., 2004). Strain variation also makes it difficult to produce reliable vaccines for prophylaxis, while increasing multidrug resistance challenges conventional treatment (Kumar et al., 2008; Han et al., 2010). Asymptomatic carriers, especially those handling food products, constitute a major threat of increased transmission (Zeng and Zhong, 2010).

Geographical information systems (GIS) have been used for surveillance and assessment of the effectiveness of typhoid prevention (Yang et al., 2007), while the establishment of time sequence models makes it possible to analyse and forecast the temporal trends (Peng et al., 2007). As it is important to develop strategies to better prevent and control typhoid fever, we used GIS and designed an analytical approach based on spatial correlation and spatial regression to study risk factors and examining tendencies of cluster formation. 


\section{Materials and methods}

\section{Study area}

Jiangsu province is located near the lower reaches of the Yangtze River in southeast P.R. China, bordering the Huanghai Sea. With an East Asian monsoon climate, the province has an annual mean temperature that generally varies between 13.6 and $16.1^{\circ} \mathrm{C}$ and an annual mean precipitation from 704 to $1,250 \mathrm{~mm}$ (Jiangsu Provincial Meteorological Bureau). The provincial population is $76,772,923$ according to the latest national census conducted in 2009.

The prefectures of Jiangsu province are geographically divided into three regions. The South comprises the prefectures Suzhou, Wuxi, Changzhou and Zhenjiang, while Xuzhou, Lianyungang, Suqian, Huai'an and Yancheng constitute the North. The prefectures of Nanjing, Yangzhou, Taizhou and Nantong make up the middle region. The water supply for the southern areas comes from the Yangtze River and the lakes, while the central region uses both the Yangtze River and the Huai River system. The northern counties depend mainly on deep wells (Han et al., 2008).

\section{Data collection}

Samples for this study were composed of typhoid fever cases (no asymptomatic carriers were included) from all Jiangsu province's 106 counties in the 13 prefectures. The data were obtained from the Jiangsu Province Center for Disease Control and Prevention (CDC) for the period 2003-2009. Demographic data from every county in Jiangsu province were collected from the Chinese National Statistics Department. Incidence rates (cases per 100,000 residents) were calculated based on the number of typhoid cases and the demographic data using the county as the primary sampling unit.

\section{Mapping and statistical methods}

ArcGIS software, version 9.3 (ESRI, Redlands, CA, USA) was used to map and analyse the distribution of typhoid cases and incidence rates by county. The average incidence rates of typhoid fever over the 7 years under study were first subjected to spatial autocorrelation analysis to denote clusters and hotspots. Various spatial correlation and regression analyses were then used to detect risk factors associated with the infection.

\section{Trend surface analysis}

The geostatistical analyst of the ArcGIS package provides a suite of statistical models and tools for exploring data variability and spatial relationships, trend surface analysis being one of them. This tool was used to describe the spatial structure and spatial change of typhoid incidence. In this way, geographical anomalies could be highlighted and scattered observations described using Global Fit procedures for three-dimensional projection of the geographical distribution of incidence. Polynomial functions were applied based on longitude and latitude including the incidence rates of the observed nodes to estimate the incidence rates of the unknown nodes. Estimations were based on random errors that involved sampling and measurement, regional trends that vary with the perspective applied and local anomalies reflecting sudden changes of incidence rates. The coefficients of the functions, calculated by the ordinary least square (OLS) technique as done by Zhou (2009) to minimize the squared surface derivations (setting expectations for local anomalies and random errors at 0 ), were derived from equations based on the residential location of the cases. Longitudes and latitudes in the maps are marked along the $\mathrm{X}$-axis and the Y-axis, respectively, as done by He et al. (2008). The $Z$ values represent the number of typhoid cases in each node of the projected grid matrix. Directional trend curves can be produced by letting the coordinates point in various directions. Two perspectives of view were selected: $0^{\circ}$ where the curves represent the trends in the east-west direction and the southnorth direction; and $45^{\circ}$, in which the directions are northeast-southwest and southeast-northwest.

\section{General spatial autocorrelation}

Autocorrelations represent the relationships of adjacent regions with respect to the same attributes (the incidence rates in this study). Areas that share similar high incidence rates were defined as autocorrelations. Considering Jiangsu province as one entity, general spatial autocorrelation analysis assumed that the counties within the province did not differ from each other. Our aim was to detect whether or not the epidemic was aggregated at the provincial level. General Moran's I was most often used for this, with the coefficient calculated as follows:

$$
\mathrm{I}=\frac{n}{\sum_{i=1}^{n} \sum_{j=1}^{n} w_{i j}} \times \frac{\sum_{i=1}^{n} \sum_{j=1}^{n} w_{i j}\left(x_{i}-\bar{x}\right)\left(x_{i}-\bar{x}\right)}{\sum_{i=1}^{n}\left(x_{i}-\bar{x}\right)^{2}}
$$


where $n$ is the numbers of counties, $\mathrm{X}_{i}$ and $\mathrm{X}_{j}$ the observations from unit $i$ to unit $j$ with regard to the phenomenon $x$ (the indicator of autocorrelations). $W_{i j}(\mathrm{~d})$ represents the adjacent weight matrix from the distance $\mathrm{d}$. If the unit $i$ for regional data is adjacent to unit $j$, then $W_{i j}(\mathrm{~d})$ is 1 , otherwise it is 0 . The $I$ index was compared with the critical value of $Z$. If $\mathrm{P}<0.05$, than Moran's $I \neq 0$, which means that the area indeed had an aggregation of typhoid infections. However, general spatial autocorrelation analysis could not discover the exact cluster locations.

\section{Local spatial autocorrelation analysis}

Given that the epidemic may vary geographically by source of infection, mode of transmission, population characteristics and behavioural distinctions, the counties may not always exhibit the same features as neighbouring counties. The results of general spatial autocorrelation, therefore, could be contrary to the results of local spatial autocorrelation. Instead of considering the province as a whole and locate the clusters exactly, local spatial autocorrelation was used to examine whether there were regional patterns. The local Moran's $I\left(L M_{i}\right)$ (equation 2) and the local G $\left(L G_{i}\right)$ (equation 3) were calculated as follows:

$$
\begin{gathered}
\mathrm{I}_{\mathrm{i}}=\frac{n^{2}}{\sum_{i} \sum_{j} w_{i j}} \times \frac{\left(x_{i}-\bar{x}\right) \sum_{i} w_{i j}\left(x_{j}-\bar{x}\right)}{\sum_{i}\left(x_{j}-\bar{x}\right)^{2}} \\
\mathrm{G}_{i}(d)=\frac{\sum_{i=1}^{n} \sum_{j=1, j \neq 1}^{n} w_{i j}(d) x_{j}}{\sum_{i=1}^{n} \sum_{j=1, j=1}^{n} x_{i}}
\end{gathered}
$$

The full range of the $L M_{i}$ and $L G_{i}$ indices were divided into five levels based on the average typhoid incidence rates for the period 2003-2009. Adapted from the natural outbreaks method as used by Zhou (2009), the partition successfully achieves the minimum differences between samples at the same level, and the maximum difference between samples at the different levels (He et al., 2008).

\section{Spatial correlation and univariate analysis}

Overlay analysis was used in spatial correlation analysis, which combined potential risk factors with the epidemic risk maps. The risk factors chosen were temperature, moisture content, elevation, transportation (railways/national highways), rivers, lakes and the nor- malized difference vegetation index (NDVI) for spring, summer, autumn and winter. We used the polygon-onpolygon overlay approach to generate the map, in which two vector polygons representing the same area and scale can be combined by uniting or cutting the polygon borders to re-establish the topological relationships between the two layers (Wang, 2006). The overlay analysis established a new database, which included all vector data for further correlation analysis. Univariate analysis was conducted with each overlay in order to examine the relationship between the incidence rates and each potential risk factor from the geographical point of view. In univariate analysis, the correlation coefficient $\mathrm{R}^{2}$ is calculated to measure the goodness-offit between the regression results and the observed data.

\section{Spatial regression and multivariate analysis}

A spatial regression model was constructed using the results of the spatial regression analysis in order to investigate the relationships between the epidemic's spatial distribution and the environmental factors mentioned above. Unlike traditional regression models, spatial models bring in stochastic effects to represent the correlations between objects instead of considering the data to be mutually independent. A logarithmic function was established, accompanied by the stochastic effects. After the establishment of the spatial regression model that includes all risk factors, variance analysis was conducted by ANOVA for the stochastic effect and coefficients for each factor to find out whether the risk factor was finally included in the model.

\section{Results}

\section{Spatio-temporal typhoid trends}

With an average of 0.57 typhoid cases per 100,000 residents, counted over the 7-year study period, Jiangsu had the second highest incidence in the East of P.R. China. The average incidence rates of the provinces in this part of the country were 0.10 (Shandong), 0.23 (Henan), 3.58 (Zhejiang) 0.29 (Anhui) and 0.29 (Shanghai) (Fig. 1). In the period 2003-2009, the total number of typhoid cases in Jiangsu province was 5,040 with the annual share starting with 2003 of 1,012, $1,271,996,669,491,374$ and 227, respectively. As can be seen in Fig. 2, the temporal distribution of cases over the 7 years were asymmetrically distributed with the epidemic decreasing in strength after 2004 and the number of cases in 2008 and 2009 accounting for only a small portion. 


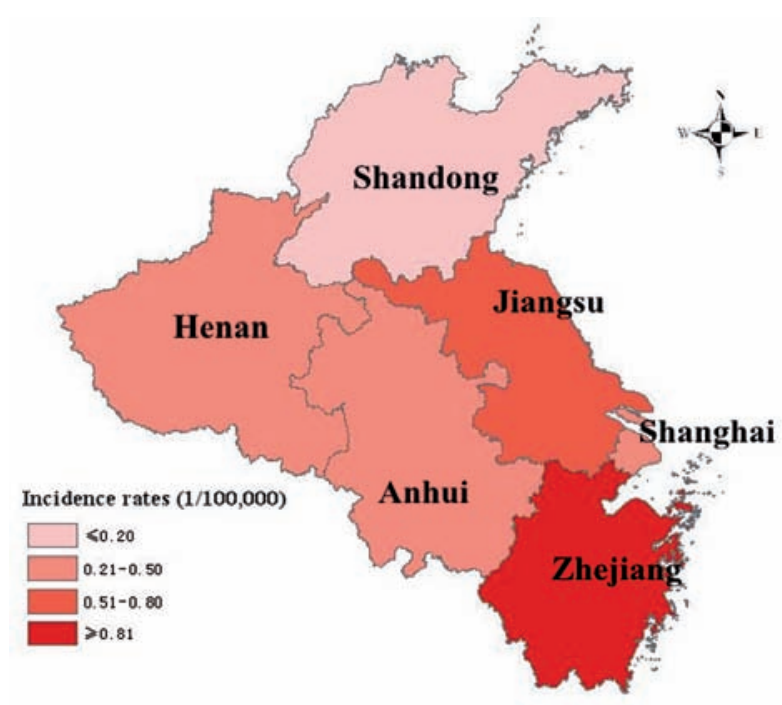

Fig. 1. Typhoid risk in Jiangsu and surrounding provinces.

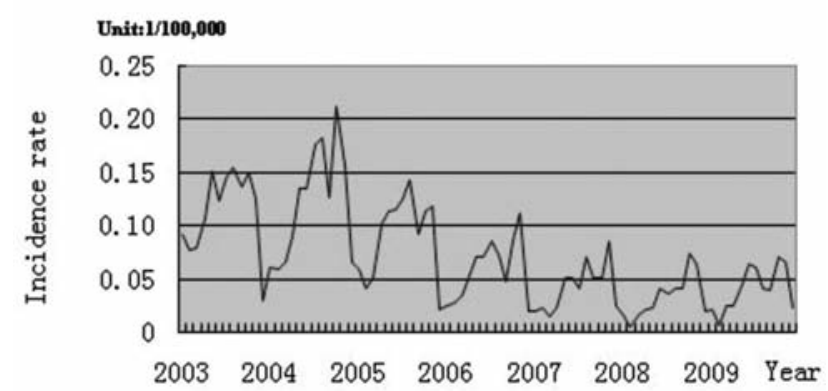

Fig. 2. The development of typhoid incidence from 2003 to 2009.

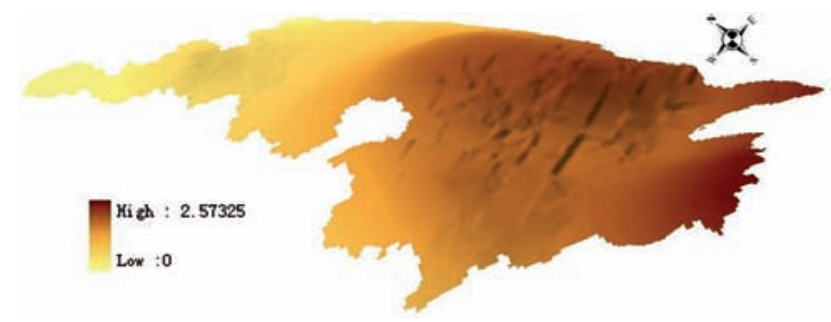

Fig. 3. Jiangsu province: smoothed incidence of typhoid fever in 2003.

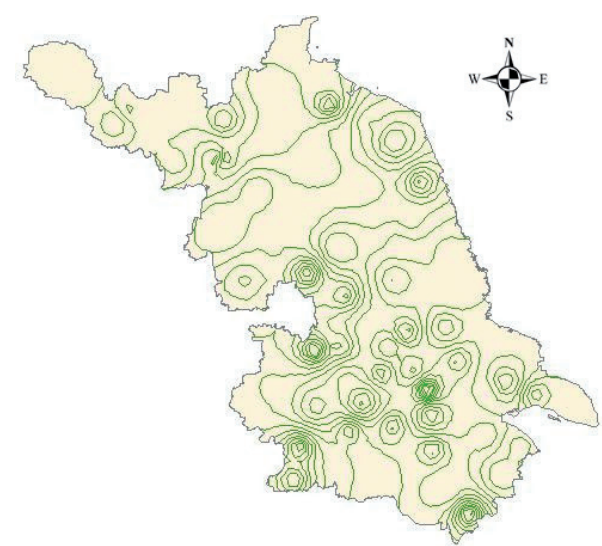

Fig. 4. Jiangsu province: contour map of typhoid incidence in 2008.
Fig. 3 shows the three-dimensional risk map of typhoid incidence rates in Jiangsu province. The darker shades indicate a higher incidence rate. Compared with the North, the South had a relatively higher incidence rate, especially in the south-eastern part. Most of the northern and the middle parts of the province showed decreasing incidence rates in the 2003-2009 period reaching lower than the average in 2008 , while some of the southern counties, including Suzhou, Kunshan and Wujiang showed increasing incidence rates 2008-2009. The contour map from 2008 (Fig. 4) confirms that the southern region had a higher incidence rate than the northern parts.

\section{Trend surface analysis}

Trend surface analysis provides a good illustration of the geographical variation of typhoid fever in the province. The list of the different directions and trends for the study period are shown in Table 1 . The trends for the 7 years investigated indicate that the typhoid incidence generally increased from west to east and from north to south. While the rate increased linearly from west to east, it grew logarithmically from north to south in 2003, 2004 and 2009. However, the rate grew faster in the North in 2003 and 2004, while it grew faster in the southern part in 2009. Turning the perspective $45^{\circ}$, the trends generally increased linearly in the north-western direction and declined toward southwest. However, the latter trend was U-shaped in 2003 and 2008-2009 indicating an increase from southwest to the middle and a decrease from the middle to northeast.

The correlation coefficient $\left(\mathrm{R}^{2}\right)$ was calculated for changes on the $\mathrm{X}$ and $\mathrm{Y}$ axes for the years 2005 to 2009 indicating the degrees-of-fit for these years (Table 2 ). The analysis showed that typhoid fever infections were more common in the southern part of the province than in the North, especially in 2008 and 2009. Fig. 5 provides graphic examples of the analysis for two of the years, 2003 and 2005. Figs. 5a and 5c show the perspective from the zero-degree, which provides the directions north to south and west to east, while Figs. 5b and $5 \mathrm{~d}$ show the $45^{\circ}$ viewpoint, which uses the northwest and southeast directions.

\section{Autocorrelation analysis}

Based on the average incidence rates, the general Moran's $I(Z=6.26, I=0.1, \mathrm{P}=0.01)$ showed significant typhoid aggregates at the provincial level. The $L M_{i}$ map (Fig. 6a) classifies the county incidence rates 
Table 1. Typhoid trends in Jiangsu province in the period 2003-2009.

\begin{tabular}{|c|c|c|c|c|}
\hline Year & West to east direction & North to south direction & Northwest to southeast & Northeast to southwest \\
\hline 2003 & Linear increase & Logarithmic growth & Linear increase & U-shaped trend \\
\hline 2004 & Linear increase & Logarithmic growth & Diminishing increase & Diminishing decrease \\
\hline 2005 & Linear increase & Linear increase & Linear increase & Linear decrease \\
\hline 2006 & Linear increase & Linear increase & Linear increase & Linear decrease \\
\hline 2007 & Linear increase & Linear increase & Linear increase & Linear decrease \\
\hline 2008 & Linear increase & U-shaped trend & Linear increase & U-shaped trend \\
\hline 2009 & Linear increase & Logarithmic growth* & Diminishing increase & U-shaped trend \\
\hline
\end{tabular}

*Particularly strong rate of increase

Table 2. Degree-of-fit for typhoid trends in Jiangsu province in the period 2005-2009.

\begin{tabular}{lccc}
\hline Year & Axis & $\mathrm{R}^{2}$ & Degree-of-fit \\
\hline 2005 & $\mathrm{X}$ & 0.094 & 6.714 \\
& $\mathrm{Y}$ & 0.550 & 39.157 \\
2006 & $\mathrm{X}$ & 0.121 & 8.946 \\
& $\mathrm{Y}$ & 0.509 & 33.139 \\
2007 & $\mathrm{X}$ & 0.247 & 21.317 \\
& $\mathrm{Y}$ & 0.650 & 120.893 \\
2008 & $\mathrm{X}$ & 0.275 & 24.633 \\
& $\mathrm{Y}$ & 0.680 & 68.151 \\
2009 & $\mathrm{X}$ & 0.304 & 28.336 \\
& $\mathrm{Y}$ & 0.727 & 85.359 \\
\hline
\end{tabular}

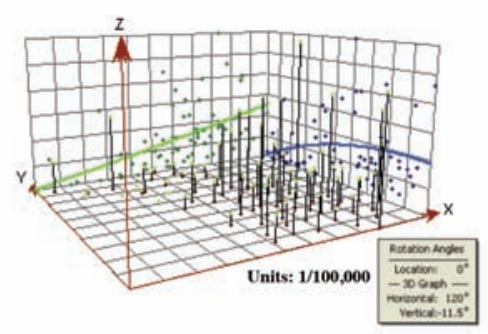

A: perspective from north to south and from west to east

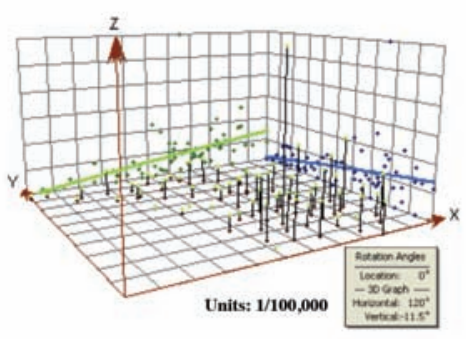

C: perspective from north to south and from west to east into five correlation levels, which are based on the optimum partition method. Significant clusters were defined as positive ("high-high" and "low-low") or negative ("high-low" and "low-high"). Positive autocorrelation refer to the higher likelihood of corresponding incidence rates at close proximity, while negative autocorrelation indicates the opposite. If the correlation level were "high-high" for a county then it would deemed to be of the high-burden type that threatens nearby counties with higher incidence rates. In the current study, four counties (Taicang, Feng, Xinqin and Xuzhou) were considered to be positively autocorrelated (Table 3). Taicang was the only "high-high" county, with statistical significance in local Getis-Ord analysis $(\mathrm{Z}=2.12, \mathrm{P}=0.03)$, while Jiangyin county

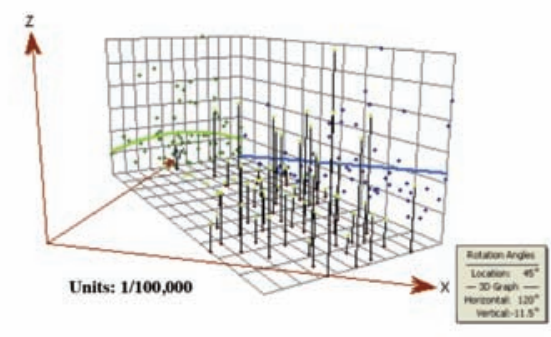

B: perspective from northwest to southeast and from northeast to southwest

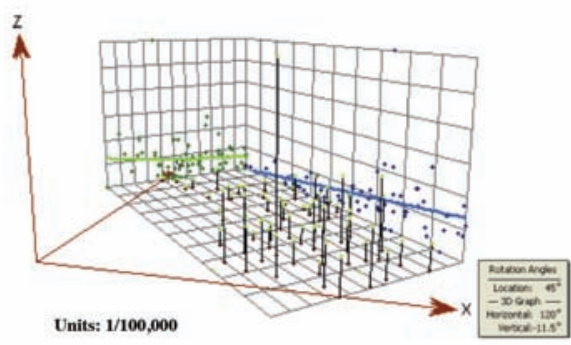

D: perspective from northwest to southeast and from northeast to southwest

Fig. 5. Trend surface analysis of typhoid fever in Jiangsu province for 2003 (A and B) and 2005 (C and D). 
Table 3. Autocorrelation analysis of five counties in Jiangsu province.

\begin{tabular}{lcccccc}
\hline County & $L M_{i}$ & $L M_{i} \mathrm{Z}$ score & $L M_{i}$ P-value & Type of correlation & $L G_{i} Z$ score & $L G_{i}$ P-value \\
\hline Taicang & $<0.001$ & 2.32 & 0.02 & High-high & 2.12 & 0.03 \\
Jiangyin & $<0.001$ & -3.25 & 0.00 & High-low & -0.47 & 0.63 \\
Feng & $<0.001$ & 2.18 & 0.03 & Low-low & 0.27 & 0.78 \\
Xinqin & $<0.001$ & 2.17 & 0.03 & Low-low & -1.97 & 0.05 \\
Xuzhou & $<0.001$ & 2.30 & 0.02 & Low-low & -2.20 & 0.03 \\
\hline
\end{tabular}

was found to be negatively correlated, i.e. a high-incidence county was surrounded by counties with low incidence rates. The $G_{i}$ map (Fig. 6b) classified the counties into five levels based on their Getis indices $\left(G_{i}\right)$. A darker shade indicates a higher index and a higher likelihood of the county being a hotspot. Taicang was detected by Getis-Ord $G_{i}$ as a statistically significant county, illustrating that this county not only had high positive correlation, but also that it was a hotspot for typhoid in Jiangsu province.

\section{Spatial correlation and univariate analysis}

Provincial overlay charts were produced for the study period (2003-2009) combining risk factors (temperature, moisture, water-bodies, highway/railway distribution, NDVI and elevation) and incidence rates (Fig. 7).

Over the 7 years, the maximum, annual mean temperature was $22.6^{\circ} \mathrm{C}$ and the minimum $14.5^{\circ} \mathrm{C}$ and the mean temperatures were highest in the southeast of part. Overall, the southern region of Jiangsu province had higher temperatures than the middle and northern parts, which corresponded to the distribution of typhoid cases. Univariate analysis determined a positive correlation between temperature and typhoid inci- dence rates $\left(\mathrm{R}^{2}=0.2, \mathrm{~F}=8.0, \mathrm{P}=0.001\right)$.

The moisture content increased smoothly from north to south reaching a highest content of $2.99 \mathrm{~g} / \mathrm{cm}^{3}$. Thus, the distribution of typhoid cases were also positively correlated with moisture $\left(\mathrm{R}^{2}=0.3, \mathrm{~F}=11.7\right.$, $\mathrm{P}<0.001)$.

With regard to water bodies, it was found that counties that were located in close proximity to a river were likely to have low incidence rates, so distance from rivers and typhoid incidence were also positively correlated $\left(\mathrm{R}^{2}=0.9, \mathrm{~F}=162.4, \mathrm{P}<0.001\right)$, In contrast, however, high typhoid incidence rates were found to be associated with proximity to lakes $\left(\mathrm{R}^{2}=1.0, \mathrm{~F}=\right.$ 4511.6, $\mathrm{P}<0.001)$. Indeed, the largest lake in Jiangsu is the Tai Lake in the far South.

Univariate analysis indicated that typhoid incidence rate was associated to highways and railways. Within a distance of approximately $16 \mathrm{~km}$, the typhoid incidence rate showed an association with highways but decreased when further away $\left(\mathrm{R}^{2}=1.0, \mathrm{~F}=10121.8, \mathrm{P}<0.001\right)$. With regard to the railways, the incidence rates showed a negative correlation within $5 \mathrm{~km}$, after which the typhoid incidence rates increased commensurate with distance $\left(\mathrm{R}^{2}=0.778, \mathrm{~F}=10.534, \mathrm{P}=0.003\right)$.

With respect to NDVI and elevation, it was found that the autumn NDVI was positively associated with
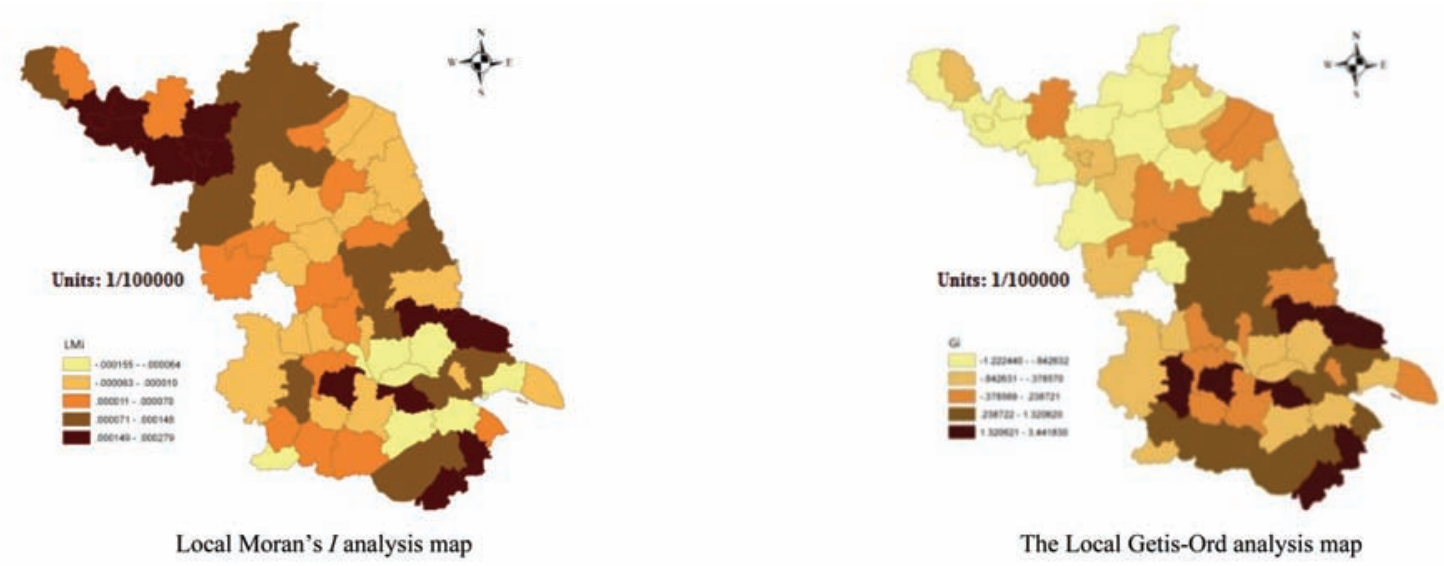

Fig. 6. Typhoid incidence in Jiangsu province: spatial distribution an local autocorrelation. The higher indices (shown by darker shades) indicate a higher degree of autocorrelation. 
A

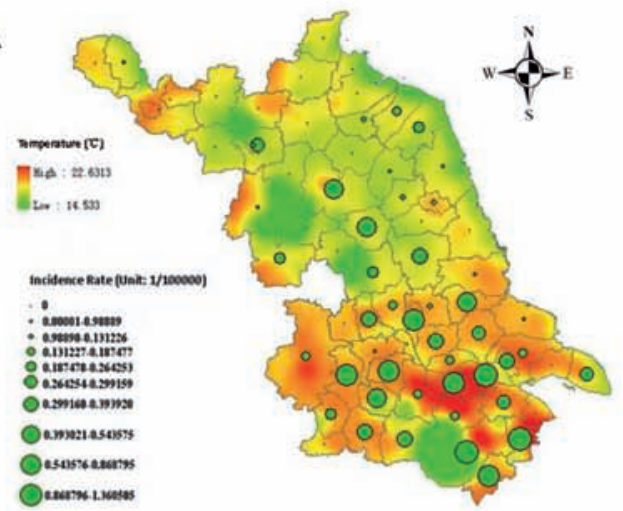

C

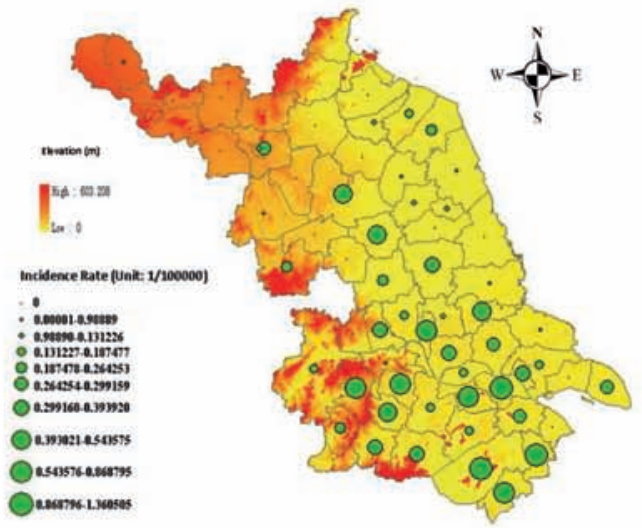

E

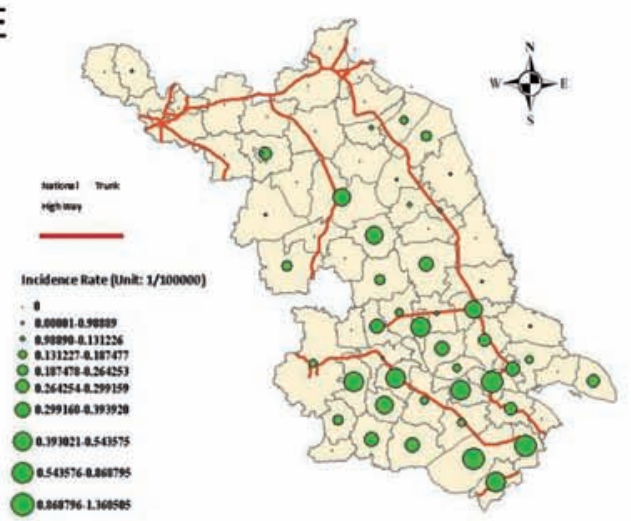

G

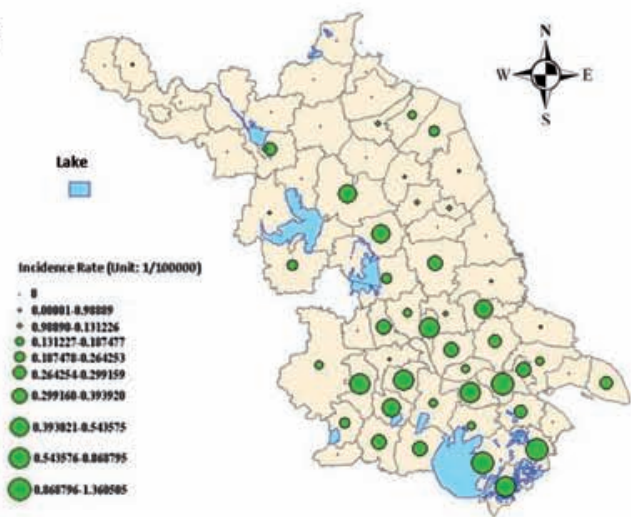

B

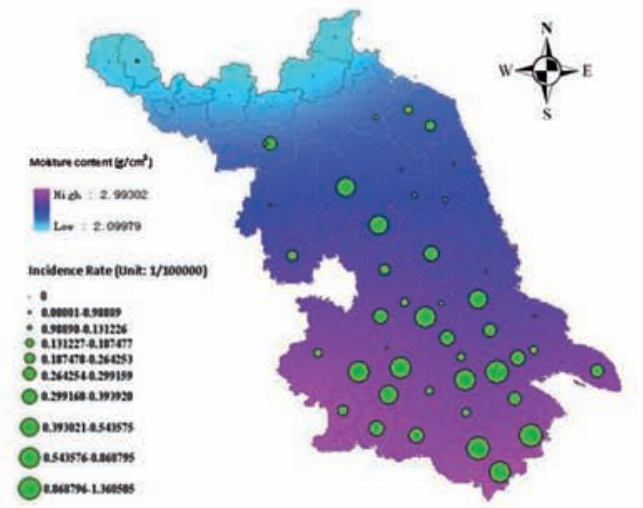

D

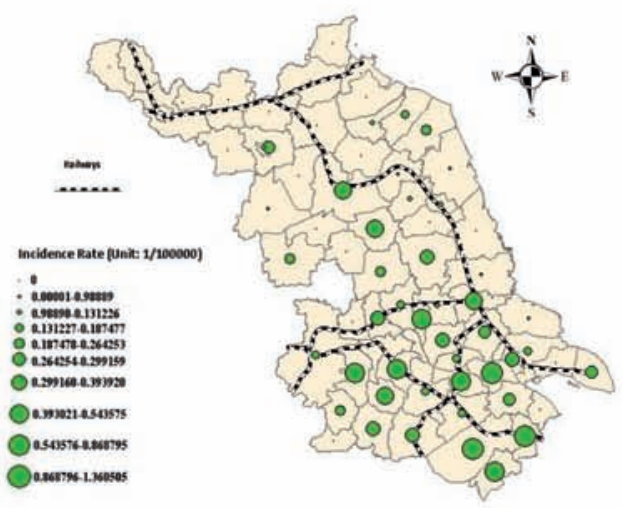

F

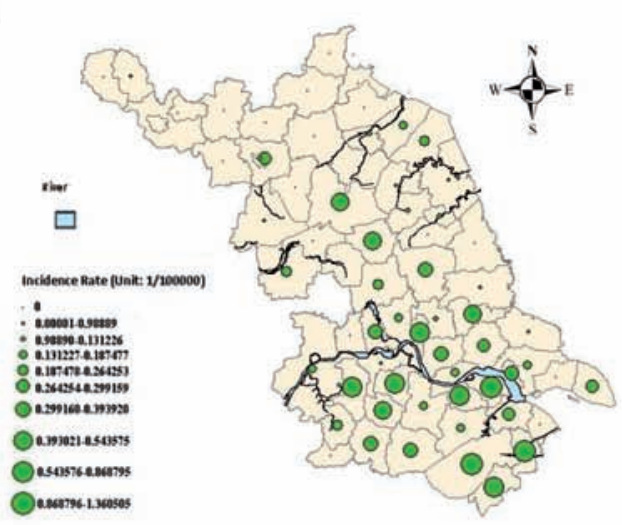

$\mathrm{H}$

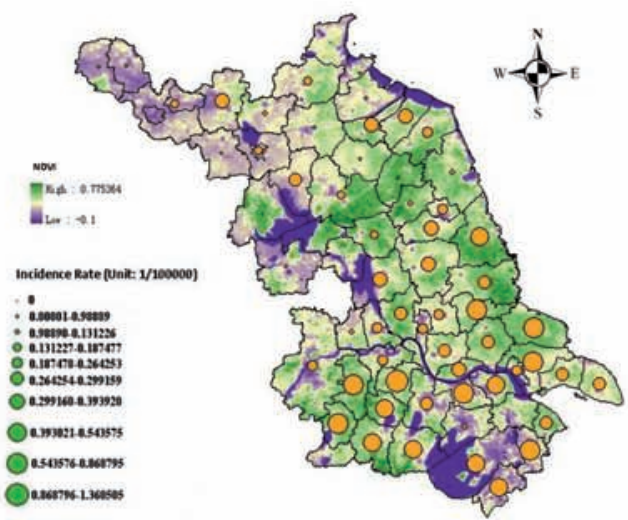

Fig. 7. Layover charts for typhoid incidence rates based on different risk factors. 
typhoid incidence $\left(\mathrm{R}^{2}=0.1, \mathrm{~F}=3.6, \mathrm{P}=0.018\right)$ but there was no noticeable correlation between elevation and typhoid incidence $\left(\mathrm{R}^{2}=0.1, \mathrm{~F}=2.2, \mathrm{P}=0.097\right)$.

\section{Spatial regression analysis and multivariate analysis}

According to the univariate analysis, incidence rate near the river was lower than further away so this variable was excluded as a risk factor in this study. In order to investigate the degree of influence from temperature, moisture content, lake proximity and autumnal NDVI, regression analysis was conducted. Concerning the correlation types of these environmental factors in univariate analysis, we chose a nonlinear regression model for the study to illustrate their multivariate affects on the epidemic. The stepwise approach was first applied to exclude the possibility of multicollinearity, a state of very high inter-associations among independent variables. The results confirmed that the four variables were not dependent in the regression process and we then applied a stepwise regression analysis by adding one risk factor at a time. As a result, four models were established with the correlation coefficients $(\mathrm{R})$ of $0.828,0.851,0.865$ and 0.866 , respectively $(\mathrm{P}<0.001)$. The degree-of-fit results showed that the model, which combined all four risk factors, had the best fit. The model was established as:

$\mathrm{y}=-0.897-0.010 \mathrm{x}_{1}+0.0071 \mathrm{x}_{1}^{2}-(8.02 \mathrm{E}-6) \mathrm{x}_{1}^{3}+$ $0.188 \mathrm{x}_{2}^{2}-0.057 \mathrm{x}_{2}^{3}+5.27 \mathrm{x}_{3}-17.1 \mathrm{x}_{3}^{2}+16.6 \mathrm{x}_{3}^{3}+$ $0.002 x_{4}^{2}-0.001 x_{3}^{4}$

where $\mathrm{y}$ represents the typhoid incidence rate (per $100,000), \mathrm{x}_{1}$ the shortest distance in $\mathrm{km}$ from the lake, $\mathrm{x}_{2}$ the moisture content in $\mathrm{g} / \mathrm{cm}^{3}, \mathrm{x}_{3}$ the NDVI and $\mathrm{x}_{4}$ the temperature in ${ }^{\circ} \mathrm{C}(\mathrm{F}=23.302, \mathrm{R}=0.886$, $\mathrm{P}<0.001)$.

\section{Discussion}

This study put emphasis on detecting how climatic factors and transportation (highways/railways) influenced typhoid incidence rates with the aim of exploring the geographical distribution patterns of this disease in Jiangsu province. Although the graphs do not represent the exact typhoid distribution, they illustrated the spread of the disease. Unlike conventional epidemiological research, spatial analysis includes the influence of geographical features on an epidemic. Not only did the GIS approach show the high incidence of typhoid in the southern region of the province, but it also denoted clusters, hotspots and other potential risk factors.

The finding that Jiangyin county is not only characterised by high incidence, but also surrounded by counties of low incidence, is not only atypical but an indication that many different factors play a role. Clearly, however, Taicang could be strongly suspected to be the focus of the typhoid epidemic since it was the only county with a hotspot active since 2008 and also had a positive autocorrelation with respect to the surrounding counties.

The incidence maps and trend surface analyses illustrate that the incidence rates were higher in the Southeast near the Yangtze River and the Tai Lake. The epidemic persisted over time in these areas characterised by a poor health (Wang and Kan, 2007). Sewage contamination of sea and lake water could make it easier for typhoid bacteria to thrive and transmit the infection (Gao and Zhang, 2008). Surface water, which is the main resource for water supplies in the South, is more easily contaminated compared with underground water from deep wells. Moreover, people in the Southeast are also known to consume uncooked seafood, which may increase the likelihood of typhoid transmission. In univariate analyses, the incidence rates decreased with proximity to rivers, while they increased with distance to lakes. This illustrates that lakes, rather than rivers, is a risk factor and indicates that the big Tai Lake might be a potential threat. Because typhoid bacteria thrive in warm, polluted water, targeting household water supply should have a great impact on typhoid transmission, particularly when coming from the lakes (Beyene et al., 2008; Anand and Ramakrishnan, 2010).

Univariate analysis of temperature and moisture content illustrated that high temperature, abundant moisture content and autumnal NDVI increase typhoid incidence. Although their influence turned out to be much less ( $\mathrm{R}^{2}$ of $0.3,0.2$ and 0.1 , respectively) than previously believed, the congenerous influence of these factors could potentially play a significant role in disease transmission. Our regression models confirm that the strength of the predictive value with respect to typhoid incidence rates is supported by environmental factors including proximity to lakes. A warmer climate could result in increased contamination of water or air and thus, indirectly influence the distribution of communicable diseases (Zhang et al., 2009). Environments characterised by warm and abundant moisture content also increase the possibility for bacterial spread as enteric bacteria such as $S$. typhi need sufficiently warm temperatures as well as abundant 
moisture to get a foothold (Kelly-Hope et al., 2008; Uneke, 2008).

Transportation factors were also found to have an impact on typhoid transmission. Since humans are the only infective source of typhoid fever, a highly developed transportation system could have brought in more transmission resources, resulting in greater chances of faecal contamination when combined with poor sanitation. Within $16 \mathrm{~km}$ from the road, typhoid fever incidence rates were strongly associated with highways. Moreover, a high correlation was also observed within $5 \mathrm{~km}$ from railways. Small restaurants, which cannot ensure sanitation and hygiene, are common at the stations along the railways and highways, where a suitable environment for the aggregation of infections is thus provided.

Potential limitations in this study include factors affecting the spatial patterns of disease, which vary in ways that are difficult to statistically analyse. Predictive models may, therefore, be somewhat different from the actual situation as pointed out by Peterson et al. (2009). In addition, our data were extracted from the official surveillance system and it is possible that counties over-report or under-report patients. It is also possible that infections had occurred but not been confirmed due to a long incubation period and thus fallen outside the study period. Indeed, patients with mild symptoms might not even have sought medical help. However, our findings constitute a first step on typhoid control and prevention, providing suggestions for intervention targets. Further evidence and clinical research are still needed to confirm all aetiological factors for typhoid fever.

\section{Conclusions}

Important environmental variables (temperature, moisture content, NDVI, proximity to lakes) and transportation factors (railways and highways) were identified as underlying the spatial distribution patterns of typhoid in Jiangsu province.

The first priority should be to provide safe water and treat sewage to avoid bacterial pollution. Sanitation at frequently visited public places, such as the areas close to highways, should be improved. Commercial kitchen workers should be trained and receive regular medical examinations in order to avoid asymptomatic carriers contaminating the food.

Screening for typhoid fever should be strengthened. Medical treatment should be given to all (including asymptomatic patients) testing positively and traceable contacts who may be carriers. Continuous sur- veillance for patients and carriers should be carried out. Health information and education on typhoid transmission routes should be provided to people in high-risk regions, while consumption of raw seafood should be discouraged.

It is vital to strengthen intervention efforts in the south-eastern region of Jiangsu, targeting the typhoid hotspot Taicang and surroundings. Clean water sources for daily use should be provided, especially in the southern counties around the Tai Lake. Prevention and control efforts should not be ignored in areas, which have experienced typhoid fluctuations in the past.

\section{Acknowledgements}

This work was supported in part by Natural Science Foundation Key Project of Jiangsu Province, P.R. China (BK2010079), "National S\&T Major Project Foundation of China” (2011ZX10004-902), Jiangsu province Health Development Project with Science and Education (NO.ZX201109), and the Priority Academic Program Development of Jiangsu Higher Education Institutions (PAPD).

\section{References}

Anand PK, Ramakrishnan R, 2010. Investigation of the outbreak of typhoid in a village of Thar Desert Rajasthan, India. Indian J Med Res 131, 199-803.

Bao CJ, Zhuang L, Song L, Zhang XF, Li XF, 2004. Surveillance and epidemiological analysis on the epidemic of typhoid and paratyphoid Salmonella during the years from 2001 2003 in Jiangsu province. Jiangsu Prev Med 15, 6-9.

Beyene G, Asrat D, Mengistu Y, Aseffa A, Wain J, 2008. Typhoid fever in Ethiopia. J Infect Develop Count 2, 448-453. Crump JA, Mintz ED, 2010. Global trends in typhoid and paratyphoid fever. Clin Infect Dis 50, 241-246.

Dong BQ, Gong J, Lin M, Yang J, 2006. Strategies on epidemiological surveillance and control of typhoid and paratyphoid in 2001-2005. Chin J Epidemiol 27, 734-735.

Dong BQ, Yang J, Wang XY, Gong J, von Seidlein L, Wang ML, Lin M, Liao HZ, Ochiai RL, Xu ZY, Jodar L, Clemens JD, 2010. Trends and disease burden of enteric fever in Guangxi province, China, 1994-2004. Bull World Health Organ 88, 689-696.

Gao JX, Zhang J, 2008. Analysis of the epidemiological characteristics and risk factors of typhoid and paratyphoid outbreak in China, 2004 to 2007. Dis Surveill 23, 698-701.

Han FA, Chen LS, Lu RZ, Jiang ZF, Yang S, 2008. Compared analysis on the quality and influence factors of rural drinking water in the northern, southern and central region of Jiangsu. Chin J Dis Control Prev 12, 441-446.

Han H, Zhou HJ, Cui ZG, Du PC, Kan B, 2010. Multilocus 
sequence typing and pulsed-field gel electrophoresis analysis of Salmonella Paratyphi A isolates from 2000 to 2008, China. Chin J Prev Med 44, 810-814.

He ZG, Han SM, Cui DY, Zhang ZJ, 2008. Discussion on statistic of spatial autocorrelation analysis. Chin J Schisto Control 20, 315-317.

Kelly-Hope LA, Alonso WJ, Thiem VD, Canh G, Anh DD, Lee H, Miller MA, 2008. Temporal trends and climatic factors associated with bacterial enteric diseases in Vietnam, 19912001. Environ Health Persp 116, 7-12.

Kumar S, Rizvi M, Berry N, 2008. Rising prevalence of enteric fever due to multidrug-resistant Salmonella: an epidemiological study. J Med Microbiol 57, 1247-1250.

Ochiai RL, Acosta CJ, Danovaro-Holliday MC, Baiqing D, Bhattacharya SK, Agtini MD, Bhutta ZA, Canh G, Ali M, Shin S, Wain J, Page AL, Albert MJ, Farrar J, Abu-Elyazeed R, Pang T, Galindo CM, von Seidlein L, Clemens JD; Domi Typhoid Study Group, 2008. A study of typhoid fever in five Asian countries: disease burden and implications for controls. Bull World Health Organ 86, 260-268.

Peng ZH, Bao CJ, Zhao Y, Yi HG, Yu H, Chen F, 2007. Application of product season model on incidence forecasting in typhoid and paratyphoid: an exploration. Chin J Dis
Control Prev 11, 560-563.

Peterson I, Borrell LN, El-Sadr W, Teklehaimanot A, 2009. A temporal-spatial analysis of malaria transmission in Adama, Ethiopia. Am J Trop Med Hyg 81, 944-949.

Uneke CJ, 2008. Concurrent malaria and typhoid fever in the tropics: the diagnostic challenges and public health implications. J Vector Dis 45, 133-142.

Wang F, 2006. Quantitative methods and applications in GIS. London: CRC Press.

Wang LQ, Kan B, 2007. Global distribution patterns of typhoid and paratyphoid fever prevention and control. Dis Surveill 22, 492-494.

Yang J, Dong BQ, Zhang J, Miao HZ, Liang DB, Wu XH, Si GA, Yang HH, Park JK, Ochiai RL, Acosta CJ, Ali M, Clemens JD, 2007. Use of GIS in assessment of typhoid fever surveillance system. Chin J Public Health 23, 1086-1088.

Zeng DW, Zhang J, 2010. Syndromic surveillance for typhoid and paratyphoid fever. Chin J Epidemiol 31, 1053-1055.

Zhang ZK, Huang YN, Huang SX, Shi ZH, Deng X, Guo CQ, Wang JJ, 2009. Model of back-propagation neural network about meteorological/geological factors and typhoid fever, paratyphoid fever. J Prev Med Inf 25, 201-203.

Zhou XN, 2009. Spatial epidemiology. Beijing: Science Press. 